\title{
Psicologia Ambiental: OBJETo, "REALIDADES" SÓCIO-FÍSICAS E VISÕES CULTURAIS DE INTERAÇÕES AMBIENTE-COMPORTAMENTO
}

\author{
Víctor Corral-Verdugo 1 \\ Universidade de Sonora - México
}

\begin{abstract}
O autor define o objeto da Psicologia Ambiental como a mútua influência de fatores ambientais e comportamentais no enfoque de problemas específicos e de suas soluções. Quanto às dimensões da realidade, discute primeiramente o tópico ambiente físico e as condições que deveriam ser levadas em conta ao estudar o comportamento ambiental e, a seguir, os aspectos sócio-culturais dos entornos e como esses influenciam as visões de mundo, tanto leigas quanto científicas, apontando que, como diferentes culturas produzem visões ambientais diferentes, estas podem se manifestar como psicologias ambientais específicas, o que não significa abandonar a idéia de uma Psicologia Ambiental universal.
\end{abstract}

Descritores: Psicologia ambiental. Objeto. Comportamento. Ambiente. Fatores socioculturais.

$\mathrm{T}$ rês importantes questões foram colocadas pelos organizadores do simpósio internacional The Role of Environmental Psychology in the study of Environmental Issues. A primeira questão enfoca o objeto da Psicologia Ambiental (i.e., 'O que a Psicologia Ambiental estuda?); a segunda tem a ver com as duas dimensões da "realidade" (i.e., os ambientes físico e sóciocultural onde os seres humanos vivem) e como eles podem ser abordados,

1 Professor da Universidade de Sonora, Hermosillo, México. Editor Associado para América Latina de Environment and Behavior. Endereço eletrônico: corral@rtn.uson.mx 
enquanto a terceira questão está interessada em discut ir se diferentes realidades sócio-culturais podem levar a diferentes abordagens em Psicologia Ambiental. A segunda e a terceira questões estão de algum modo relacionadas, desde que ambas consideram o aspecto do ambiente sócio-cultural como instigador de ou como afetado por um comportamento ambiental relevante.

Assim, ao tentar responder à segunda questão, enfocarei o tópico ambiente físico e as condições que deveríamos levar em conta ao estudar o comportamento ambiental. Então, ao tentar responder a terceira questão, dirigirei a minha atenção aos aspectos sócio-culturais de nossos entornos, e como esses aspectos influenciam as nossas visões de mundo, leiga e científica.

\section{O objeto da Psicologia Ambiental}

Ao enfocarmos o tópico do objeto da Psicologia Ambiental, é conveniente tornar claro que a Psicologia Ambiental é uma área ou sub-disciplina de uma ciência psicológica mais geral (Holahan, 1982; Sommer, 2000). A este respeito, McKenzie-Mohr e Oskamp (1995, p. 7) mencionam que a Psicologia Ambiental está contida "na Psicologia [como] o sub-campo da psicologia ambiental". Como uma área da psicologia, a Psicologia Ambiental tem também como seu objeto o estudo do comportamento e de seus correlatos (Clitheroe, Stokols, \& Zmuidzinas, 1998). É claro que, como no caso de outras áreas da psicologia, a Psicologia Ambiental tem os suas próprias especificidades e interesses. Neste caso, a Psicologia Ambiental está envolvida com os modos pelos quais os aspectos social e físico do ambiente influenciam o comportamento das pessoas e como as ações das pessoas, por sua vez, afetam os seus entornos. Este envolvimento torna necessária a promoção de esforços interdisciplinares a fim de abranger uma variedade diversa de dimensões (social, material) influenciadas por ou afetando o comportamento.

O objeto da Psicologia Ambiental tem sido discutido em termos de paradigmas definidores (Bechtel, 1996), níveis de análise de comportamento ambientalmente relevante (Altman \& Rogoff, 1987), ou escopo inter/intra disciplinar da Psicologia Ambiental (Veitch \& Arkkelin, 1995). Contudo, 
neste artigo, o foco da discussão é o modo pelo qual duas abordagens dominantes no estudo das relações comportamento-pessoas definem o que é importante para os psicólogos ambientais. Uma preocupação particular é a de que a divergência entre essas duas abordagens poderiam, não apenas estar produzindo uma deformação do objeto da Psicologia Ambiental mas, além disto, poderiam estar levando ao aparecimento de duas "psicologias ambientais" separadas, contribuindo para a confusão, para as ambiguidades na definição da Psicologia Ambiental e, provavelmente, para uma duplicação dos esforços de pesquisa nesta área.

A Psicologia Ambiental emergiu como uma área aplicada da psicologia objetivando resolver problemas com respeito às interações ambientecomportamento. Como é bem conhecido, duas abordagens dominaram a área (e ainda prevalecem) desde as suas origens. Uma abordagem privilegiou o estudo dos efeitos ambientais sobre o comportamento. Estudos referentes à percepção ambiental, mapas cognitivos, preferências ambientais, ao efeito da estimulação ambiental sobre o desempenho humano, às relações entre o projeto e o uso de espaços construídos, e às avaliações pós-ocupação são representativos desta abordagem (Evans \& Cohen, 1987; Kaplan, 1987; Lynch, 1960; Veitch \& Arkkelin, 1995; Zimring \& Reizenstein, 1980). Esta tradição em pesquisa pode ser identificada como a abordagem ambiente $\rightarrow$ comportamento $(\mathrm{a} \rightarrow \mathrm{c})$.

A segunda tradição hospeda os estudos referentes a como e porquê o comportamento humano afeta o ambiente. Esta inclui a pesquisa sobre conservação e comportamento sustentável, o estudo de crenças ambientais, valores, personalidade e capacidades (entre outros) e a investigação da associação entre variáveis demográficas e comportamento ambientalmente relevante. Igualmente sob esta perspectiva está a relação entre educação ambiental e comunicação, fatores situacionais (tanto normativos quanto físicos) e conservação ambiental (De Young, 1996; Dunlap \& Van Liere, 1978; Hines, Hungerford, \& Tomera, 1987; Scott, 1999; Zelezny, 1999). Podemos identificar esta perspectiva como a abordagem comportamento $\rightarrow$ ambiente $(\mathrm{c} \rightarrow \mathrm{a})$.

Estas duas abordagens co-existiram como tradições de pesquisa na Psicologia Ambiental desde sempre. Contudo, a interação entre elas é antes 
exceção do que regra. Embora a maioria dos investigadores reconheçam que o ambiente é afetado pelas ações e disposições humanas e, simultaneamente, que esses aspectos psicológicos são influenciados por fatores ambientais, as relações ambiente-comportamento são, na sua maior parte, estudadas de um modo fragmentado.

Ao estudar o efeito do comportamento humano sobre o ambiente mtural $(\mathrm{a} \rightarrow \mathrm{c})$, a maioria dos pesquisadores pensa ser necessário investigar como fatores contextuais influenciam a promoção de comportamentos próambientais $(\mathrm{c} \rightarrow \mathrm{a})$. Por exemplo, sabe-se que alguns fatores ambientais físicos, tais como a escassez de recursos naturais, aumentam a motivação para a conservação, o que, por sua vez, afeta positivamente o status do ambiente (Corral-Verdugo, 2002). Sabe-se, também, que uma experiência prévia de contatos com o ambiente promove uma afinidade emocional para com a natureza, o que, consequentemente, promove a sua conservação (Chipeniuk, 1995; Kals \& Maes, 2002). Mais um caso: a percepção de desperdício de recursos por outras pessoas influencia negativamente o próprio comportamento de conservação (Corral-Verdugo, Frías, Pérez-Urías, Orduña, \& Espinoza, 2002; De Oliver, 1999). Em outras palavras, ao se estudar o efeito do comportamento sobre o ambiente, é igualmente necessário estudar quais influências ambientais são promotoras significativas de comportamento ambiental responsável, e assim por diante.

Outros exemplos ilustram como os pesquisadores poderiam e deveriam combinar aspectos da percepção ambiental e de várias influências contextuais com o efeito do comportamento sobre o ambiente. Um desses exemplos involve a noção de affordances de Gibson (1977), que descreve como propriedades estimuladoras do ambiente levam as pessoas a mostrar respostas efetivas. De acordo com Gibson, algumas características dos estímulos ambientais dispõem a ou encorajam ações adaptativas ou soluções de problemas. Desde que alguns problemas ambientais requerem soluções comportamentais (Suárez, 1998), a investigação de affordances que levam os seres humanos a resolver problemas ambientais é uma idéia pertinente. Steele (1980) e Corral-Verdugo (1994) sugeriram que tal perspectiva é uma explicação plausível de ações pró-ambientais, e chamam esses comporta- 
mentos de solução de problemas de competências ambientais. O último propõe que tais affordances podem ser consideradas como requisições próambientais, que têm origens contextuais, seja físicas ou normativas. Affordances ou requisições, tais como escassez de recursos, conveniência de comportamento, valores e normas ambientais, são instigadores significativos de competência ambiental (Corral-Verdugo, 2002). E, mais ainda: a competência ambiental é auto-percebida, gerando a "motivação da competência" - um sentimento auto-recompensador resultante de ser competente (De Young, 1996) - o qual, por sua vez, promove mais comportamentos de conservação (CorralVerdugo, 1996). A cadeia de efeitos mútuos ambiente $\leftrightarrow$ comportamento pode ser representada como se segue:

\begin{tabular}{|ccccccc|}
\hline Ambiente & $\rightarrow$ & Comportamento & $\rightarrow$ Ambiente & $\rightarrow$ Comportamento & $\rightarrow$ & Ambiente \\
$\begin{array}{c}\text { Requisições } \\
\&\end{array}$ & $\rightarrow$ Ação pró-ambiental & $\rightarrow$ Conservação & $\rightarrow$ & Competência & $\rightarrow$ & Mais \\
affordances & (competência) & (percebida) & motivação & & conservação \\
\hline
\end{tabular}

Portanto, o objeto da Psicologia Ambiental é a influência mútua de fatores ambientais e comportamentais, ao tentar focalizar problemas específicos e soluções. A ênfase desta definição na resolução de problemas revela a natureza aplicada da Psicologia Ambiental. Contudo, isto não limita o seu escopo. Em adição à sua natureza aplicada, a Psicologia Ambiental gera pesquisa básica. O próprio conceito de affordances, tanto quanto as noções de competência de motivação, requisições pró-ambientais, transações ambiente-comportamento etc., emergiram da orientação de pesquisa básica da Psicologia Ambiental.

"Influência mútua" significa que, a todo momento, o ambiente afeta o modo como percebemos, sentimos, e agimos a fatores contextuais físicos e/ou normativos, e que aquelas percepções, sentimentos e ações afetam os componentes sócio-físicos do ambiente. Infelizmente, a divergência entre as abordagens ambiente $\rightarrow$ comportamento e comportamento $\rightarrow$ ambiente ainda prevalece e pode levar ao estabelecimento de duas "psicologias ambientais" separadas. Uma delas é chamada, por alguns autores, de "psicologia arquitetural" (Canter, 2002), e a outra é reconhecida como "psicologia da 
conservação" (Shipee, 1980). Com respeito à última, pelo menos um grupo virtual international. ${ }^{2}$ Discutiu a conveniência de sua "abertura" como uma nova área da psicologia. Esta nova área, presumivelmente independente da Psicologia Ambiental (Brook, 2001; Myers, 2001), estudaria a influência do comportamento sobre o nosso entorno, visto os crescentes problemas ambientais. Embora alguns psicólogos ambientais vejam esta tendência - concebida como uma "especialização" - como um movimento lógico, e talvez necessário, outros pensam que a separação da psicologia da conservação da Psicologia Ambiental não é conveniente (ver Myers, 2001). Ter duas abordagens complementares trabalhando independentemente significa um desperdício de esforço de pesquisa. Além disto, esta separação contribuiria para um objeto mais difuso da PA (i.e., o estudo das relações unidirecionais ambiente-comportamento).

Isto conduz à discussão de quão pertinente é o estabelecimento da Psicologia da Conservação como uma sub-área especializada da Psicologia Ambiental ou como um campo independente da Psicologia. Face aos raciocínios acima propostos, a procura de uma interação mais alta entre as abordagens $\mathrm{a} \rightarrow \mathrm{c} \mathrm{e} \mathrm{c} \rightarrow \mathrm{a}$ deveria levar à consideração da Psicologia da Conservação como uma Psicologia Ambiental especializada, que teria o objetivo de estudar determinantes e consequências do comportamento ambiental responsável. Contudo, se a falta de interação entre aquelas abordagens continuar, é muito provável que a Psicologia da Conservação assuma uma independência real (ou, pelo menos, formal) ante a Psicologia Arquitetural. Esta opção implica na emergência de duas novas áreas na Psicologia (Psicologias Arquitetural e da Conservação).

\section{Dimensões da "realidade"}

Dois aspectos principais da realidade constituem o objeto da Psicologia Ambiental. De um lado, há um ambiente objetivo, tangível, feito de elementos

2 Conservation Psychology: conservation-psychology@ listserver.itd.umich.edu 
físico-químicos, que afetam as nossas sensações, percepções e ações (Gibson, 1977). Nosso comportamneto também afeta esta dimensão física da realidade, modificando e alterando a sua composição. De outro lado, os seres humanos, como sujeitos sociais, criam e também estão expostos a um ambiente de artefatos culturais, símbolos e convenções. $\mathrm{O}$ ambiente criado-pelo-homem da cultura, normas, regras e leis estimula nossos organismos. As características resultantes são propriedades "convencionadas" do ambiente, onde os estímulos são classificados segundo as atribuições geradas por um grupo social (Segall, Dasen, Berry, \& Poortinga, 1999). Assim, estes dois aspectos da realidade (material, cultural) constituem o ambiente sócio-físico.

O ambiente é composto pelas dimensões espacial e temporal (além das propriedades relativistas de Einstein, as quais, em termos práticos da vida diária humana, são irrelevantes dado não afetarem a nossa percepção do mundo). Objetos estimulantes encontram-se em coordenadas tridimensionais e eventos ambientais ocorrem em um continuum temporal. Uma combinação de referências espaciais produz a forma, o contraste e outros aspectos visualmente percebidos de objetos no ambiente.

Além disto, os objetos são percebidos e reconhecidos em termos de outras propriedades físicas. Alguns liberam compostos químicos que afetam o nosso paladar e olfato. Quando vibram ou se movem, muitos são capazes de induzir respostas auditivas; sua composição atômica, como objetos sólidos, líquidos ou gasosos, macios ou ásperos, afetam sistemas táteis. Igualmente, quase todos os objetos poderiam ferir o tecido corporal, causando dor. O próprio organismo é uma fonte de estimulação proprioceptiva, cinestésica e vestibular. Este tipo de estimulação está baseada em propriedades físico-químicas dos objetos. O ambiente físico é um importante aspecto da realidade, que se impinge sobre os seres humanos, e que influencia as suas cognições, sentimentos e ações.

Gibson (1977) foi um proponente do "realismo ambiental", a noção de que a nossa percepção ambiental e conhecimento derivam de um contato direto com objetos ambientais. Também Altman e Rogoff (1987) incluiram o ambiente físico como um aspecto essencial do objeto psico-ambiental. 
Segundo Werner, Brown e Altman (2002, p. 204), “o ambiente físico é mais do que um fundo. Ele muda e se move, e suas muitas formas proporcionam barreiras, desafios e oportunidades para os participantes". Neste nível, o estudo da relação ambiente $\rightarrow$ comportamento focaliza o modo como os estímulos físico-químicos e os contextos ambientais afetam as percepções, as preferências, o conhecimento e as ações.

As facetas humanas do comportamento, como discutido anteriormente, também afetam o ambiente físico de modo que a interação humanocomportamento tem implicações sobre o status da dimensão químico-física da realidade. Nossas ações produziram mudanças significativas na composição da atmosfera (Dunn \& Flavin, 2002), solo e florestas (Abramovitz \& Mattoon, 1999; World Resource Institute, 1992), qualidade e disponibilidade da água (Gardner, 2002; O'Meara, 1999), recursos naturais inorgânicos (Flavin \& Dunn, 1999) e biodiversidade (Brown \& Flavin, 1999). Assim, a Psicologia Ambiental tem muito a ver com o estudo dos aspectos físicos da realidade mais geral. Procurar modos apropriados de estudar as relações comportamento - ambiente físico deveria ser um dos interesses dos psicólogos ambientais.

Como poderíamos estudar as relações ambiente-comportamento com respeito a cada um dos aspectos da realidade? Os psicólogos ambientais constróem cenários de pesquisa. Embora esses cenários não estejam mais em laboratórios fechados (i.e., contextos isolados e controlados onde o pesquisador manipula variáveis independentes), o cenário da pesquisa tem se tornado um contexto social. Ao coletar os dados, usualmente o pesquisador de Psicologia Ambiental coloca os seus participantes ante estímulos que não correspondem aos da interação ambiente-comportamento estudada. Os estímulos que eliciam as respostas dos participantes são o próprio pesquisador e suas requisições, enquanto o contexto verbal substitui o ambiente físico supostamente estudado pelo pesquisador (Daniel \& Ittelson, 1981). Segundo Evans (1995), o estudo da Psicologia Ambiental na ausência do ambiente físico é, mais precisamente, um estudo de Psicologia Social. 
Por exemplo, ao interagir de um modo pró-ambiental, a pessoa maneja objetos (usualmente recursos naturais), que constituem os estímulos ante os quais ele ou ela responde ao conservar o ambiente. Alguns autores reivind icam que a avaliação das ações pró-ambentais deveria ser conduzida colocando os participantes ante esses estímulos físicos, de modo que a correspondência entre o comportamento de conservação e o registro de tal comportamento aumente. Tal situação produziria avaliações mais válidas do comportamento ambiental. A fim de ilustrar esta situação, Corral-Verdugo (1997) pediu a participantes de um estudo sobre comportamento de reutilização e reciclagem para indicar quantos objetos haviam sido reutilizados e reciclados, tendo aqueles objetos diante deles. Ao fazer isto, os seus relatos foram mais precisos do que aqueles produzidos no contexto social de auto-relatos do comportamento.

Obviamente, os comportamentos ambientais ocorrem em cenários sociais (que usualmente incluem os aspectos físicos, como referido anteriormente). Por exemplo, ações pró-ambientais algumas vezes aparecem no contexto de normas, valores e crenças ambientais. Isto deveria levar o investigador a considerar aqueles contextos como eliciadores pertinentes das respostas dos participantes em um dado estudo. Portanto, ao planejar um estudo, o pesquisador deveria definir os cenários social e físico correspondentes aos comportamentos sob escrutínio.

\section{Visões culturais das interações humano-comportamento}

Diferentes culturas induzem as pessoas a sustentar diferentes visões de mundo e também afetam o modo como o cientista pensa as relações pessoasambiente. A ciência - incluindo os seus ramos sociais - é um empreendimento cultural Assim, é construída de convenções que emergem de pessoas específicas em tempos e lugares específicos. Por exemplo, Dunlap (1980) sugere que, por muitos anos, o Paradigma Social Dominante (PSD) considerava os seres humanos como independentes da natureza e sua fé como dominando a natureza. Esta crença cultural, característica das sociedades ocidentais, era 
tão forte que a ciência social a aceitava como o seu paradigma (Arcury, Johnson, \& Scollay, 1986). Catton e Dunlap (1980) denominam esta influência sobre a ciência de Paradigma da Exceção Humana (PEH).

As visões culturais também afetam as noções leigas das relações humano-ambiente. Por exemplo, as sociedades ocidentais parecem ser mais dualistas em seu pensamento sobre o papel dos seres humanos na Natureza, enquanto as culturas tradicionais, não ocidentais, são mais holísticas. A distinção natureza-cultura é um exemplo típico das "duas espécies diferentes de realidade formadas no amanhecer da idade moderna", como Colwell (1997) ressalta. Um estudo de Bechtel, Corral-Verdugo e Pinheiro (1999) mostrou essa diferença cultural. Eles utilizaram a escala do "Novo Paradigma Ambiental", que avalia as crenças antropocêntricas (i.e., os seres humanos estão acima da natureza e daí não têm de considerá-la na medida em que consomem os seus recursos) e ecocêntricas (i.e., as pessoas são uma parte da natureza e devem considerá-la no uso dos recursos). Os resultados mostraram que os norte-americanos consideram a visão antropocêntrica como extremamente oposta à ecocêntrica, enquanto os brasileiros não vêem uma contradição entre essas duas visões. Corral-Verdugo e Armendáriz (2000) também encontraram esta visão holística entre os participantes mexicanos, o que lhes sugeriu que, nas sociedades da América Latina, "os indivíduos podem estar ligados ao equilíbrio natural e, ao mesmo tempo, acreditar no controle humano sobre a natureza e estar interessados em possíveis lucros que possam obter da natureza" (p. 30). Esses autores lembram que, coincidentemente, esta é uma descrição da doutrina do desenvolvimento sustentável, que procura um equilíbrio entre a proteção do ambiente e a satisfação das necessidades humanas (Meadows, Meadows, \& Randers, 1992), que, hoje em dia, raramente é praticada na maior parte das sociedades.

As diferenças culturais e as especificidades dos problemas sociais e ambientais também produzem explicações particulares do comportamento humano em uma cultura específica. Esta declaração é suportada pelos promotores da assim chamada Indigenous Psychology ou Psicologia Indígena ou Autóctone. Indigenous Psychology é a compreensão de que o comportamento humano está enraizado em um contexto cultural particular (Kim, 
1990). Segundo Triandis (2000), uma vantagem da Indigenous Psychologyé que se pode descobrir fenômenos que só existem em uma cultura, e são inteiramente desconhecidos e não esperados por pesquisadores de outras culturas. Se isto for verdade, a visão de mundo e o comportamento ambiental variam em função de psicologias indígenas particulares.

A Psicologia indígena dominante no mundo é a Psicologia euroamericana (Triandis, 1997), que tem como premissas sócio-culturais as tradições do individualismo, do liberalismo e da racionalidade (Yang, 2000). Como vimos, o dualismo é também uma importante característica das culturas e da psicologia ocidentais. Os indivíduos, nas culturas ocidentais, devem atingir suas metas (esperadas) participando de práticas baseadas nestas tradições. Isto explica porque a psicologia ocidental está tão interessada em explicar os determinantes do comportamento ambiental estudando as disposições individuais (habilidades tecnológicas, motivos egoistas, locus de controle, auto-estima), manisfestações de liberalismo (orientação política, estilos democrático/autoritário), e processos racionais (conhecimento, estratégias de solução de problemas).

Essas premissas euro-americanas não podem ser generalizadas para o mundo inteiro. Na Ásia Oriental, por exemplo, "o individualismo e a racionalidade são vistos como instáveis, enquanto os apegos emocionais e de relações são considerados estáveis" (Kim, 2000, p. 279). Como em algumas sociedades lationoamericanas (Bechtel, Corral-Verdugo, \& Pinheiro, 1999; Corral-Verdugo \& Armendáriz, 2000), na Ásia, a visão da relação naturezacultura é mais holista do que dualista. Os seres humanos são considerados como parte da natureza, e a harmonia é o valor mais importante pois ela integra os seres humanos à natureza, aos espíritos e a outros indivíduos (Kim, 2000). Ao contrário da explicação psicológica ocidental, a psicologia indígena oriental está baseada nas tradições de relações, de emoções e do coletivismo. Desde que as psicologias indígenas da Ásia oriental, assim como as africanas e as latinoamericanas, são relativamente novas, elas não abordaram empiricamente o problema das relações ambiente-comportamento usando as suas perspectivas particulares. Para mim, seria de enorme interesse conhecer como as psicologias não-ocidentais abordam o problema da 
deterioração ambiental. Por exemplo, será o apego emocional à natureza e à própria comunidade um determinante significativo de conservação ambie ntal? Será a inteligência social (antes do que a inteligência tecnológica) uma condição para cuidar dos recursos naturais? Será a participação comunitária na resolução de problemas ambientais uma estratégia mais efetiva do que a soma dos comprometimentos e ações individuais? Estas, e outras interessantes questões, seriam colocadas por estas explicações indígenas alternativas.

Outro aspecto relacionado à cultura é o fator econômico. Os psicólogos ambientais, nas sociedades não-industrializadas, estão mais interessados em problemas sócio-econômicos que caracterizam suas sociedades: pobreza, questões de justiça ambiental, doença e má qualidade de vida, escassez de recursos naturais (Adeola, 1996; Corral-Verdugo, 2002; Wiesenfeld \& Sánchez, 2002). Esses aspectos têm um impacto em suas noções e práticas de sustentabilidade. Por exemplo, é compreensível que a re-utilização e a austeridade sejam mais praticadas em sociedade mais pobres (Corral-Verdugo, 1996, 1997), enquanto a reciclagem é a preferida nas sociedades industrializadas. Enquanto os pesquisadores nos países mais ricos vêem o controle populacional como uma das ações pró-ambientais mais importantes (a ser praticada pelas nações mais pobres), as sociedades sub-desenvolvidas vêem a austeridade e a justiça social (a ser praticada pelas sociedades mais ricas) como uma condição para a sustentabilidade.

Portanto, há a necessidade de incorporar a cultura na explicação das relações ambiente-comportamento. Necessitamos estudar o modo como a cultura influencia as visões de mundo, normas, sentimentos e comportamentos das pessoas. Desde que diferentes culturas produzem visões ambientais diferentes, estas podem se manifestar como psicologias ambientais específicas. Isto não significa que temos de abandonar a idéia de uma Psicologia Ambiental universal. Há mais aspectos comuns entre pessoas humanas do que diferenças, e deveríamos encontrar como estes aspectos diversos e similares participam na nossa própria adaptação ao nosso ambiente, de uma maneira eficiente e responsável. 
Corral-Verdugo, V. (2005). Environmental psychology: object, sociophysical "realities" and cultural views of environment-behavior interactions. Psicologia USP, 16(1/2), 71-87.

\begin{abstract}
The author defines the object of Environmental Psychology as the mutual influence of environmental and behavioral factors on the analysis of specific problems and their solutions. As for the dimensions of reality, he firstly discusses the topic physical environment and the conditions that should be taken into account when one studies environmental behavior. Secondly, he discusses the socio-cultural aspects of our surroundings and how they influence both our lay and scientific views of the world, arguing that, as different cultures produce different environmental views, these can manifest in specific Environmental Psychologies, but this does not mean the idea of a universal Environmental Psychology should be abandoned.
\end{abstract}

Index terms: Environmental psychology. Object. Behavior. Environment. Sociocultural factors.

Corral-Verdugo, V. (2005). Psychologie de l'environnement: Objet, "realites" socio-physiques et visions culturelles d'interactions environnement-comportement. Psicologia USP, 16(1/2), 71-87.

Résumé: L'auteur définit l'objet de la Psychologie de l'Environnement comme étant l'influence mutuelle de facteurs environnementaux et comportementaux dans l'optique de problèmes spécifiques et de leurs solutions. Quant aux dimensions de la réalité, il discute, en premier lieu, le thème environnement physique et les conditions dont il faudrait ternir compte en étudiant le comportement environnemental et, ensuite, les aspects socioculturels de notre entourage et comment ceux-ci influent sur nos visions du monde, profanes aussi bien que scientifiques, soulignant que, différentes cultures produisant des visions environnementales différentes, celles-ci peuvent se manifester en tant que Psychologies de l'Environnement spécifiques, ce qui ne signifie pas abandonner l'idée d'une Psychologie de l'Environnement universelle.

Mots-clés: Psychologie de l'environnement. Objet. Comportement. Environnement. Aspects socioculturels. 


\section{Referências}

Abramovitz, J. N., \& Mattoon, A. T. (1999). Reorienting the forest products economy. In L. Starke (Ed.), State of the world. A Worldwatch Institute Report on Progress Toward a Sustainable Society. New York: W. W. Norton \& Company.

Adeola, F. O. (1996). Environmental contamination, public hygiene, and human health concern in the third world: the case of Nigerian environmentalism. Environment \& Behavior, 28, 614-646.

Altman, I., \& Rogoff, B. (1987). World views in psychology: Trait interactional, organismic, and trsansactional perspectives. In D. Stokols \& I. Altman (Eds.), Handbook of environmental psychology. New York: Wiley.

Arcury, T., Johnson, T., \& Scollay, S. (1986). Ecological worldview and environmental knowledge: The "New Environmental Paradigm". Journal of Environmental Education, 17, 35-40.

Bechtel, R. B. (1996). The paradigm of environmental psychology. American Psychologist, 51, 1187-1188.

Bechtel, R. B., Corral-Verdugo, V., \& Pinheiro, J. Q. (1999). Environmental belief systems. United States, Brazil, and Mexico. Journal of Crosscultural Psychology, 30, 122-128.

Brook, A. T. (2001). What is "Conservation Psychology?". Population and Environmental Psychology Bulletin, 27(2), 1-2.

Brown, L. R., \& Flavin, C. (1999). A new economy for a new century. In L. Starke (Ed.), State of the World. A Worldwatch Institute Report on Progress Toward a Sustainable Society. New York: W.W. Norton \& Company.

Canter, D. (2002). Editorial reflections. Journal of Environmental Psychology, 22, 1-3.

Catton, W. R., \& Dunlap, R. E. (1980). A new ecological paradigm for post-exuberant sociology. American Behavioral Scientist, 24, 15-47.

Chipeniuk, R. (1995). Childhood foraging as a means of acquiring competent cognition about biodiversity. Environment \& Behavior, 27, 490-512.

Clitheroe, H. C., Stokols, D., \& Zmuidzinas, M. (1998). Conceptualizing the concept of environment and behavior. Journal of Environmental Psychology, 18, 103-112.

Colwell, T. (1997). The nature-culture distinction and the future of environmental education. Journal of Environmental Education, 28, 4-8.

Corral-Verdugo, V. (1994). ¿Mapas cognoscitivos o competencias ambientales? [Cognitive maps or environmental competencies?]. Acta Comportamentalia, 2, 25-55. 
Corral-Verdugo, V. (1996). A structural model of reuse and recycling in Mexico. Environment \& Behavior, 28, 665-696.

Corral-Verdugo, V. (1997). Dual 'realities' of conservation behavior: Self-reports vs observations of reuse and recycling behavior. Journal of Environmental Psychology, 17, 135-145.

Corral-Verdugo, V. (2002). A structural model of pro-environmental competency. Environment \& Behavior, 34, 531-549.

Corral-Verdugo, V., \& Armendariz, L. I. (2000). The "New Environmental Paradigm" in a Mexican community. Journal of Environmental Education, 31, 25-31.

Corral-Verdugo, V., Frías, M., Pérez, F., Orduño, V., \& Espinoza, N. (2002). Residential water consumption, motivation for conserving water, and the continuing tragedy of the commons. Environmental Management, 30, 527-535.

Daniel, T., \& Ittelson, W. (1981). Conditions for environmental research: Reactions to Ward and Russell. Journal of Experimental Psychology: General, 110, 153-157.

De Oliver, M. (1999). Attitudes and inaction. A case study of the manifest demographics of urban water conservation. Environment \& Behavior, 31, 372-394.

De Young, R. (1996). Some psychological aspects of a reduced consumption lifestyle: The role of intrinsic satisfaction and competence motivation. Environment \& Behavior, 28, 358-409.

Dunlap, R. E. (1980). Paradigmatic change in social science: From human excemptionalism to an ecological paradigm. American Behavioral Science, 24, 5-14.

Dunlap, R. E., \& Van Liere, K. D. (1978). The new environmental paradigm. Journal of Environmental Education, 9, 10-19.

Dunn, S., \& Flavin, C. (2002). Moving the climate agenda forward. In Worldwatch Institute (Ed.), State of the World 2002. New York: W.W. Norton \& Company.

Evans, G. (1995). Measurement of the environment as stressor. Unpublished manuscript. Cornell University. Ithaca, New York.

Evans, G., \& Cohen, S. (1987). Environmental stress. In D. Stokols \& I. Altman (Eds.), Handbook of environmental psychology. New York: Wiley.

Flavin, C., \& Dunn, S. (1999). Reinventing the energy system. In L. Starke (Ed.), State of the World. A Worldwatch Institute Report on Progress Toward a Sustainable Society. New York: W.W. Norton \& Company.

Gardner, G. (2002). The challenge for Johannesburg: Creating a more secure world. In Worldwatch Institute (Ed.), State of the World 2002. New York: W.W. Norton \& Company. 


\section{Víctor Corral-Verdugo}

Gibson, J. J. (1977). The theory of affordances. In R. Shaw \& J. Bransford (Eds.), Perceiving, acting, and knowing. Hillsdale, NJ: Lawrence Erlbaum.

Hines, J. M., Hungerford, H. R., \& Tomera, A. N. (1987). Analysis and synthesis of research on responsible environmental behavior: A meta-analysis. Journal of Environmental Education, 18, 1-8.

Holahan, C. J. (1982). Environmental psychology. New York: Random House.

Kaplan, B. (1987). Aesthetics, affect, and cognition: Environmental preferences from an evolutionary perspective. Environment \& Behavior, 19, 3-32.

Kals, E., \& Maes, J. (2002). Sustainable development and emotions. In P. Schmuck \& W. Schultz (Eds.), Psychology of sustainable development. Norwell, MA: Kluwer.

Kim, U. (1990). Indigenous psychology: Science and applications. In R.W. Brislin (Ed.), Applied cross-cultural psychology. Newbury Park, CA: Sage.

Kim, U. (2000). Indigenous, cultural and cross-cultural psychology: A theoretical, conceptual, and epistemological analysis. Asian Journal of Social Psychology, 3, 265-287.

Lynch, K. (1960). Image of the City. Cambridge, MA: MIT Press.

McKenzie-Mohr, D., \& Oskamp, S. (1995). Psychology and sustainability: An introduction. Journal of Social Issues, 51, 1-14.

Meadows, D. H., Meadows, D. L., \& Randers, J. (1992). Beyond the limits: Confronting global collapse, envisioning a sustainable future. Post Mills, VT: Chelsea Green.

Myers, G. (2002). Some issues to consider in the role of psychology in conservation. Population and Environmental Psychology Bulletin, 27(2), 2-4.

O'Meara, M. (1999). Exploring a new vision for cities. In L. Starke (Ed.), State of the World. A Worldwatch Institute Report on Progress Toward a Sustainable Society. Nueva York: W.W. Norton \& Company.

Scott, D. (1999). Equal opportunity, unequal results. Determinants of household recycling intensity. Environment \& Behavior, 31, 267-290.

Segall, M. H., Dasen, P., Berry, J. W., \& Poortinga, Y. H. (1999). Human behavior in global perspective (2nd ed.). Boston: Allyn \& Bacon.

Shippee, G. (1980). Energy consumption and conservation psychology: A review and conceptual analysis. Environmental Management, 4, 297-314.

Sommer, R. (2000). Discipline and field of study: A search for clarification. Journal of Environmental Psychology, 20, 1-4. 
Steele, F. (1980). Defining and developing environmental competence. In C. P. Alderfer \& C. L. Cooper (Eds.), Advances in experimental social processes. Vol. 2, pp. 225244.

Suárez, E. (1998). Problemas ambientales y soluciones conductuales. In J. I. Aragonés \& M. Amérigo (Eds.), Psicología ambiental. Madrid: Ediciones Pirámide.

Triandis, C. (1997). Cross-cultural perspectives on personality. In R. Hogan, J. Johnson \& S. Briggs (Eds.), Handbook of personality psychology. San Diego, CA: Academic Press.

Triandis, C. (2000). Dialectics between cultural and crosscultural psychology. Asian Journal of Social Psychology, 3, 185-195.

Veitch, R., \& Arkkelin, D. (1995). Environmental psychology: An interdisciplinary perspective. Englewood Cliffs, NJ: Prentice-Hall.

Yang, K. S. (2000). Monocultural and cross-cultural indigenous approaches: The royal road to the development of a balanced global psychology. Asian Journal of Social Psychology, 3, 241-263.

Werner, C., Brown, B., \& Altman, I. (2002). Transactionally oriented research: Examples and strategies. In R. B. Bechtel \& A. Churchman (Eds.), Handbook of environmental psychology. New York: Wiley.

Wiesenfeld, E., \& Sánchez, E. (2002). Sustained participation: A community based approach to addressing environmental problems. In R. Bechtel \& A. Churchman (Eds.), Handbook of environmental psychology. New York: Wiley.

World Resources Institute (1992). World Resources 1992-93. New York: Oxford University Press.

Zelezny, L. C. (1999). Educational interventions that improve environmental behaviors: A meta-analysis. Journal of Environmental Education, 31, 5-14.

Zimring, C. M., \& Reizenstein, J. E. (1980). Post-occupancy evaluation. An overview. Environment \& Behavior, 12, 429-450.

Recebido em 5.04.2004

Revisto e encaminhado em 23.02.2005

Aceito em: 7.03.2005 